\title{
The hardening hypothesis: does it matter?
}

\section{Graeme Docherty, Ann McNeill}

Tobacco control has made great progress in reducing smoking prevalence in countries where cigarette smoking rates were once high, as in the UK. ${ }^{1}$ When prevalence began to fall at a slower rate from the late 1980 s, a number of commentators suggested this may be due to the remaining smokers becoming more hardened' to cessation, that is, less likely to be influenced by cessation measures. ${ }^{2}$ To assess this hypothesis was therefore seen as important because if true, it could be argued that more investment should be made to target the 'hardened' groups as existing interventions were clearly not affecting these smokers; but if false, then it could be assumed that existing interventions were largely adequate.

An assessment of hardening is incomplete without defining what constitutes a hardcore (or 'hardened') smoker. Definitions include having no intention to quit, usually lack of previous quit attempts and a measure of dependence. ${ }^{3}$ In general, most authors agree that hardcore smokers are those who do not want to quit or those who find it very difficult to quit; in each case, smokers who are very likely to continue smoking.

One study found support for hardening. ${ }^{4}$ This research used the Fagerstrom Test of Nicotine Dependence (FTND) as an indicator of hardening, analysing FTND scores in countries with varying smoking prevalence and finding that dependence scores were higher in countries with lower smoking rates, although their selection of datasets has come under criticism. ${ }^{5}$ Additionally, data from cessation trials have shown quit rates have decreased over time. ${ }^{6}$ However, these trials excluded those who did not want to quit, clearly an important subgroup. Even if dependence in treatment seekers is

Division of Epidemiology and Public Health, UK Centre for Tobacco Control Studies, University of Nottingham, Nottingham, UK

Correspondence to Graeme Docherty, Division of Epidemiology and Public Health, UK Centre for Tobacco Control Studies, C100, Clinical Sciences Building 2, University of Nottingham, City Hospital, Hucknall Road, Nottingham NG5 1PB, UK:

graeme.docherty@nottingham.ac.uk increasing, this does not imply the same is occurring in the general smoker population and therefore may not give the full picture.

Studies of hardcore prevalence have now been conducted in several industrial countries $^{7-12}$ showing a range from $0.03 \%$ to nearly $16 \%$. The lack of a standard definition as well as the cross-sectional nature of the datasets used makes comparisons across countries and over time difficult, and unsurprisingly prevalence tends to be higher where definitions are more flexible. The most recently published study is from Norway, ${ }^{12}$ where the authors measured hardcore prevalence using an annual survey and found prevalence decreased between 1996 and 2009. Current research on UK nationally representative data by the UK Centre for Tobacco Control Studies appears to support the Norwegian findings. One drawback of this research is the frequent inclusion of average daily cigarette consumption (CPD) in the hardcore definition. CPD has fallen in some countries with well developed tobacco control measures, ${ }^{13}$ possibly as a result of taxation measures and the introduction of smokefree laws; but, it has been argued that this does not necessarily mean tobacco dependence is decreasing because CPD is not well correlated with nicotine intake. ${ }^{13}$

Many of the prevalence studies also assessed the sociodemographics of hardcore smokers and found that they are more likely to be from a more deprived socioeconomic background. ${ }^{12}$ Inequalities and smoking are closely related. In the UK, we are concerned that smoking has reduced substantially among the more affluent, while prevalence has changed very little in some disadvantaged groups. ${ }^{14}$ The relationship between smoking and hardship in the UK is strong and consistent whatever indicator of disadvantage is used; for example, those with a known mental health condition have much higher smoking rates than the general population. ${ }^{14}$ A study from Australia confirmed hardcore smokers are more likely to be susceptible to a mental health condition but found this did not appear to be increasing over time, which again is evidence against hardening. ${ }^{16}$
Even if the hardening hypothesis can be discounted, this does not mean that we do not need to think carefully about what we are doing for those who cannot or will not quit. It is clear that if we are serious about reducing health inequalities, we need to improve our efforts to reduce smoking among the disadvantaged in society. We therefore suggest an additional, more targeted approach. General measures such as taxation, smoke-free laws and the removal of tobacco branding are all necessary to emphasise the unique dangers of smoking and these need to be sustained and enhanced. These measures encourage quit attempts as well as discourage uptake generally. ${ }^{17}$ However, such measures will not do enough to narrow the health gap between the rich and the poor. Currently, the UK offers comprehensive treatment to all smokers, which is free to the most deprived, and it has been demonstrated that these services are reaching more deprived smokers. ${ }^{18}$ However, our research indicates that there are still some disadvantaged groups who are not being helped by the services, some of whom may be resistant to authorities and fearful of what they perceive as judgemental health professionals. Additional approaches, including community outreach and harm reduction methods, are therefore being explored. These approaches are likely to be more expensive than the general measures outlined above.

Discussions of hardening and the consequences are most relevant to countries in the later stages of the tobacco epidemic, ${ }^{19}$ where prevalence was once high and has since fallen, but unequally across the sociodemographic groups. These are countries most likely to have implemented general tobacco control measures and in a position to explore further ways of helping hard to reach groups. Such discussions are less applicable to nations with historically low prevalence, or current very high prevalence, and/or where infrastructure and economic circumstances cannot support more intensive approaches. Indeed, the recent Framework Convention on Tobacco Control (FCTC) guidelines for cessation recommended a stepwise approach in the context of other FCTC articles which motivate smokers to quit, outlining which interventions to prioritise given the limited resources and building on a country's infrastructure, taking limited resources into account. ${ }^{20}$

We remain of the view, however, that tobacco control and health professionals should be assisting all smokers, not just those who are easiest to reach. We believe that this approach is ethically prudent. 
From an academic point of view, we believe there is merit in future research exploring and improving our understanding and measurement of hardcore smoking, ${ }^{21}$ but implementing evidencebased comprehensive strategies alongside a more targeted approach to protect the most disadvantaged in society seems to us to be warranted whatever the outcomes of such research.

Funding The authors are fully or partly funded by the UK Centre for Tobacco Control Studies, a UK Clinical Research Collaboration (UKCRC) initiative with funding from the British Heart Foundation, Cancer Research UK, Economic \& Social Research Council, Medical Research Council and the National Institute for Health Research, under the auspices of the UKCRC. Grant Number RES-590-28-0004.

Competing interests None

Contributors GD and AM are the authors of this paper.

Provenance and peer review Commissioned; externally peer reviewed.

Tobacco Control 2012;21:267-268.

doi:10.1136/tobaccocontrol-2011-050382

\section{REFERENCES}

1. Cancer Research UK. Smoking Statistics. http:// info.cancerresearchuk.org/cancerstats/types/lung/ smoking/ (accessed 24 Sep 2011)

2. Coambs RB, Kozlowski LT, Ferrence RG. The Future of tobacco use and smoking research. In: Ney T,
Gale A, eds. Smoking and Human Behaviour. New York: Wiley \& Sons Ltd, 1989:337-48.

3. Costa ML, Cohen JE, Chaiton M, et al. 'Hardcore' definitions and their application to a populationbased sample of smokers. Nicotine Tob Res 2010;12:860-4.

4. Fagerstrom K, Furberg H. A comparison of the Fagerstrom Test for Nicotine Dependence and smoking prevalence across countries. Addiction 2008; 103:841-5.

5. Etter JF. Hardening the methods, a comment on Fagerstrom \& Furberg (letter). Addiction 2008; 103:1575-7

6. Irvin JE, Brandon TH. The increasing recalcitrance of smokers in clinical trials. Nicotine Tob Res 2000;2:79-84.

7. Emery S, Gilpin EA, Ake C, et al. Characterising and identifying 'hard-core' smokers: implications for further reducing smoking prevalence. Am J Public Health 2000:90:387-94.

8. Jarvis $\mathbf{M}$, Wardle $\mathrm{J}$, Waller $\mathrm{J}$, et al. Prevalence of hardcore smoking in England, and associated attitudes and beliefs: cross-sectional study. BMJ 2003:326:1061-6.

9. Macintosh H, Coleman T. Characteristics and prevalence of hardcore smokers attending UK general practitioners. BMC Fam Pract 2006;7:24.

10. Ferketich AK, Gallus S, Colombo P, et al. Hardcore smoking among Italian men and women. Eur J Cancer Prev 2009;18:100-5

11. Sorg A, Xu J, Doppalapudi SB, et al. Hardcore smokers in a challenging tobacco environment: the case of Missouri. Tob Control 2011;20 $388-90$.

12. Lund M, Lund KE, Kvaavik E. Hardcore smokers in Norway 1996-2009. Nicotine Tob Res 2011:13:1132-9.
13. Warner KE, Burns DM. Hardening and the hardcore smoker: concepts, evidence and implications. Nicotine Tob Res 2003;5:37-48.

14. Jarvis $\mathbf{M}$, Wardle J. Social patterning of individual health behaviours: the case of cigarette Smoking. In: Marmot M, Wikinson R, eds. Social Determinants of Health. Oxford: Oxford University Press, 2006.

15. McManus S. Cigarette Smoking and Mental Health Analysis of Data from the Adult Psychiatric Morbidity Survey. 2007. http://www.natcen.ac.uk/study/ cigarette-smoking -mental-health laccessed 12 0ct 2011).

16. Matthews R, Hall WD, Gartner CE. Is there evidence of 'hardening' among Australian smokers between 1997 and 2007? Analyses of the Australian National surveys of mental health and well-being. Aust New Zeal J Psychiatr 2010;44 1132-6.

17. Chaiton MO, Cohen JE, Frank J. Population health and the hardcore smoker: Geoffrey Ward revisited. $J$ Public Health Policy 2008;29:307-18.

18. Bauld L, Judge K, Platt S. Assessing the impact of smoking cessation services on reducing health inequalities in England: observational study. Tob Control 2007; 16:400-4.

19. Lopez AD, Collinshaw NE, Piha T. A descriptive model of the cigarette epidemic in developed countries. Tob Control 1994:3:242-7.

20. Raw M. Framework Convention on Tobacco Control (FCTC) Article 14 guidelines: a new era for tobacco dependence treatment. Addiction 2011:106:2055-7.

21. Hughes $\mathbf{J}$. The hardening hypothesis: is the ability to quit decreasing due to increasing nicotine dependence? A review and commentary. Drug Alcohol Depend 2011;117:111-17. 